\title{
SHARED CONSUMPTION:
}

A Technological analysis

by

John A. Weymark

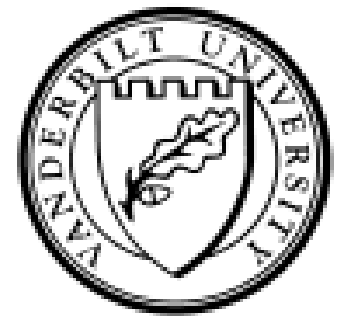

Working Paper No. 03-W01R

February 2003

Revised July 2003

DEPARTMENT OF ECONOMICS

VANDERBILT UNIVERSITY

NASHVILLE, TN 37235

www.vanderbilt.edu/econ 


\title{
Shared Consumption: A Technological Analysis*
}

\author{
John A. Weymark \\ Department of Economics, Box 1819, Station B, \\ Vanderbilt University, Nashville, TN 37235, U.S.A. \\ (e-mail: john.weymark@vanderbilt.edu)
}

February 2003

Revised, July 2003

\begin{abstract}
*This article is a substantially revised version of the first chapter of my doctoral dissertation [Weymark (1977)]. I am grateful to Myrna Wooders for suggesting that this research is still of interest and for encouraging me to revise it for publication. The first two chapters of my thesis formed the basis of my presentation to the European Science Foundation Workshop on "Local Public Goods, Politics and Multijurisidictional Economies" held at the Université Paris 1 (Panthéon-Sorbonne) in July 2002. I have benefitted from comments received from Jean-Marc Bonnisseau, John Conley, and an anonymous referee. I am also grateful to Karl Shell and Bob Inman for their comments on the earlier thesis version of this article and to the Canada Council for supporting my thesis research.
\end{abstract}




\begin{abstract}
James Buchanan (Economica, 1966) has argued that Alfred Marshall's theory of jointly-supplied goods can be extended to analyze the allocation of impure public goods. This article introduces a way of modelling sharing technologies for jointly-supplied goods that captures the essential features of Buchanan's proposal. Public and private goods are special cases of shared goods obtained by appropriately specifying the sharing technology. Necessary conditions for an allocation in a shared goods economy to be Pareto optimal are identified and related to the optimality conditions for public and private goods.
\end{abstract}

Keywords and Phrases: impure public goods, shared goods, Pareto optimality.

\title{
JEL Classification Number: H41
}




\section{Introduction}

Early critics of Samuelson's (1954) model of pure public goods argued that it is difficult to find examples of goods that exhibit the complete nonrivalness in consumption that Samuelson's definition requires. For example, Margolis (1955) suggested that national defence and lighthouses exhibit this nonrivalness, but most other goods that are collectively consumed, such as highways and the courts, are subject to congestion. Alternative models of impure public goods abound, of which Tiebout's (1956) theory of local public goods and Buchanan's (1965) theory of clubs have been the most influential. ${ }^{1}$ With these kinds of goods, each member of the community or club consumes the same quantity of the good, but individual utilities also depend on the number of people that the good is being shared with.

For many publicly-provided goods, decisions about the location or other characteristics of the good affect the distribution of benefits. For example, consider a police force of a given size in a city with two geographically distinct precincts. More police allocated to one precinct will increase safety there at the expense of the safety of those individuals in the second precinct. Typically, the increase in safety of one group does not diminish the safety of the other group by the same amount because the police can respond to crime throughout the city. Similarly, shifting more police into the investigation of burglary from the traffic enforcement unit will differentially affect the benefits received from the police force by different residents. There are many other examples of goods that are not completely rival in consumption and for which the mix of benefits can be varied. For example, the benefits an individual receives from a fire station depend on the distance from, and hence the location of, a fire station, and the benefits of a mosquito eradication programme depend on where the repellant is released. ${ }^{2}$

Buchanan (1966, 1967, 1968) has suggested that Marshall's (1920) theory of joint supply can be usefully extended to analyze these kinds of impure public goods. With Marshallian joint supply, the produced good embodies multiple final products. For example, wool and mutton are the final products embodied in sheep. Buchanan extended this idea to impure public goods by regarding the products of an impure public good as being the individual-

\footnotetext{
${ }^{1}$ See Cornes and Sandler (1996) for a discussion of the literature on club goods and local public goods.

${ }^{2}$ The police example is adapted from Shoup (1964) and the other examples from Buchanan (1968).
} 
specific consumptions obtained from this good. Because more than one individual can benefit from their provision, I refer to such goods as shared goods. ${ }^{3}$ The feasible distributions of consumptions are described by a sharing technology. A sharing technology is simply a production function that specifies the distributions of consumption that are possible as a function of the quantity of the shared good that is available. ${ }^{4}$

Buchanan distinguished between the units in which a shared good is measured (production units) and the units in which the consumption of the services of this good are measured (consumption units). In the mosquito eradication example, the quantity of the good is measured by the quantity of repellant released and consumption is measured by the reduction in the number of mosquitos in a particular locality. In the case of crime protection, production of the good could be measured by the size of the police force, while consumption could be measured in terms of an index of safety.

For Marshallian goods, as the scale of production is increased, the quantities of the final products increase proportionally because no variability in the product mix is possible. For many shared goods, it is possible to vary both the scale of shared good provision and the mix of final consumptions. For example, in the fire protection example, once the location of the fire hall is fixed, while everyone can be better protected by hiring more firemen, the relative proportions of fire protection to the residents of the area are fixed. However, in contrast to Marshallian jointly-supplied goods, the proportions in which benefits are received can also be varied by choosing a different location for the fire hall. ${ }^{5}$

\footnotetext{
${ }^{3}$ I have borrowed this terminology from Inman (1971), who applies it to a particular class of congestible goods.

${ }^{4}$ Production theory has also been used in a number of other ways to model impure public goods. Oakland $(1972,1987)$ considered goods, such as highways, for which an individual's benefits depend on his usage and a measure of congestion, where congestion is a function of capacity and the total usage by all individuals. See also the related model in Cornes and Sandler (1996, Chapter 12). Inman (1971) proposed a model in which the services from a public facility depend on the size of the facility and the number of users. The properties of Inman's model have been investigated in some detail by Hillman (1978). Sandmo (1973) used a production function in which final consumption is a function of the quantities of a private and public good, as is the case when a private car is needed to take advantage of a publicly-provided highway. In Cornes and Sandler (1996, Chapter 8), an impure public good is a Marshallian good with two final products, one private and one public.

${ }^{5} \mathrm{~A}$ further contrast between shared goods and Marshallian goods is that the products of a Marshallian good can be traded, whereas the consumptions associated with shared goods
} 
By appropriate specifications of the sharing technology, both private goods and pure public goods are obtained as special cases. In view of this fact and the fact that a wide range of examples of impure public goods can be modelled as shared goods, it is somewhat surprising that shared goods à la Buchanan have received virtually no attention in the subsequent literature. ${ }^{6}$ For example, Buchanan's work on shared goods is not mentioned in Cornes and Sandler's (1996) monograph on externalities, public goods, and club goods. Oakland (1972), in his survey of the theory of public goods, simply dismissed Buchanan's theory (see footnote 5), without describing the theory in any detail. Plott and Meyer (1975) described what a sharing technology is and showed how pure public goods and private goods are special cases, but did little more than argue for the usefulness of this way of modelling impure public goods.

The description and analysis of shared good provision in Buchanan's work is quite sketchy and somewhat informal. In this article, I propose a way of modelling sharing technologies for jointly-supplied goods that captures the essential features of Buchanan's contribution. I use this model to identify the necessary conditions for an allocation to be Pareto optimal in an economy with shared goods. I also show how the shared goods optimality conditions can be used to obtain the standard optimality conditions for private and public goods. My analysis of these optimality conditions complements and extends the corresponding analysis in Buchanan (1966, 1968).

My model of an economy with shared goods is described in Section 2. Optimality conditions for this economy are derived and discussed in Section 3. In order to obtain the optimality conditions for the limiting case of a pure public good, the model is reformulated in Section 4 in terms of consumption shares. Some concluding remarks are presented in Section 5.

\section{The Model}

There are two types of good in the economy, private and shared. The set of private goods is $\mathcal{K}=\{1, \ldots, K\}$ and the set of shared goods is $\mathcal{M}=$ $\{1, \ldots, M\}$. In order to distinguish between the consumption and production

cannot. Buchanan (1966, pp. 406-407) explicitly noted this point, which is something that was overlooked when Oakland (1987, p. 490) criticized Buchanan and others for treating public good provision as an instance of joint production.

${ }^{6}$ An exception is Weymark (1979), where Buchanan's framework was used to explore the relationship between the optimality conditions for public and private goods. 
of a shared good, the produced good is called a shared good facility. Shared goods facilities are produced using private goods as inputs by firms. There is no joint production of shared goods. The aggregate production possibilities set for the $m$ th shared good facility is $Y^{m}{ }^{7}$ It is assumed that $Y^{m}$ is a closed convex set and that it is not possible to produce any output without a positive amount of at least one private good. It is also assumed that the output of this shared good facility is freely disposable. The set of feasible production vectors in $Y^{m}$ can be described using a production function $Y^{m}(\cdot)$. A production vector $\left(\mathbf{z}_{m}, y_{m}\right)$ is feasible if

$$
Y^{m}\left(\mathbf{z}_{m}, y_{m}\right) \leq 0
$$

where $y_{m}$ is the output of this shared good facility and $\mathbf{z}_{m}=\left(z_{m 1}, \ldots, z_{m K}\right)$ is the nonnegative vector of private goods used to produce this facility. The production function $Y^{m}(\cdot)$ is assumed to be continuously differentiable.

In addition to producing shared goods facilities, the firms in this economy also produce private goods. An aggregate production set $F$ is used to describe this sector of the economy. $F$ is assumed to be closed, convex, and to satisfy free disposability. It is also assumed that no good can be produced without the use of some of the other private goods as inputs. Letting $F(\cdot)$ denote the corresponding production function, $F$ is the set of net output vectors $\mathbf{w}=\left(w_{1}, \ldots, w_{K}\right)$ for which

$$
F(\mathbf{w}) \leq 0
$$

where for each good $k \in \mathcal{K}, w_{k}$ is positive (resp. negative) if it is an output of (resp. input to) this sector. $F(\cdot)$ is assumed to be continuously differentiable.

The producers of shared goods need not be the agents who make them available to consumers. For example, roads and fire halls are typically built by private firms, but are supplied to the public by governments. In order to allow for a range of alternative provision arrangements, I refer to the intermediaries between the producers and consumers of shared goods as groups. A group could be a government, a club in the sense of Buchanan (1965), or even a single individual. The latter case would apply if there is private provision of a shared good. The set of groups is $\mathcal{G}=\{1, \ldots, G\}$. It is possible that groups have overlapping memberships.

\footnotetext{
${ }^{7}$ Because the focus of the analysis is on the sharing technology, for simplicity, the production sectors of the economy are only described in terms of aggregate production technologies, not the technologies of individual firms.
} 
In terms of the allocation of shared goods facilities between groups, these goods exhibit the rivalness of private goods. Hence, for each $m \in \mathcal{M}$,

$$
\sum_{g=1}^{G} y_{g m} \leq y_{m},
$$

where $y_{g m}$ is the amount of the $m$ th shared good facility owned by the $g$ th group.

In principle, any group could provide any of the $M$ shared goods to consumers. For simplicity, I assume that each group has a separate sharing technology for each shared good and that each shared good facility only provides one kind of consumption benefit to consumers. The set of consumers is $\mathcal{I}=\{1, \ldots, I\}$ and the consumption by the $i$ th consumer of the $m$ th shared good as a result of its provision by the $g$ th group is $s_{i g m}$. As described in Section 1, the quantities of these goods are measured in consumption units, whereas the facilities themselves are measured in production units. The $g$ th group's sharing technology for the $m$ th shared good is $S^{g m}$. An element of $S^{g m}$ is a vector $\left(\mathbf{s}_{g m}, y_{g m}\right)$, where $\mathbf{s}_{g m}=\left(s_{1 g m}, \ldots, s_{I g m}\right) . S^{g m}$ is assumed to be a closed convex set and the individual consumptions are assumed to be freely disposable. If $y_{g m}=0$, then $s_{i g m}=0$ for all $i$ as well. Letting $S^{g m}(\cdot)$ denote the corresponding sharing function, $S^{g m}$ is the set of vectors $\left(\mathbf{s}_{g m}, y_{g m}\right)$ for which

$$
S^{g m}\left(\mathbf{s}_{g m}, y_{g m}\right) \leq 0 \text {. }
$$

The free disposability assumption implies that if the distribution of consumptions $\mathbf{s}_{g m}$ is feasible with a facility of size $y_{g m}$, then it is possible to exclude the $i$ th consumer from some or all of the units of consumption $s_{\text {igm }}$ in this distribution. Whether or not such exclusion is feasible is of fundamental importance when considering the incentives a group or individual has for providing a shared good. However, in this article, I am only concerned with optimal provision of shared goods. Because it is never optimal to exclude an individual from consuming a good with positive benefits if this can be done without cost and without affecting the consumption of the other individuals, the assumption that $S^{g m}$ satisfies free disposal is innocuous here. ${ }^{8}$

A sharing technology is illustrated in Figure 1. The curve shows all the efficient distributions of consumptions for two consumers from a shared

\footnotetext{
${ }^{8}$ If there is any rivalness in the consumption of a shared good, it may well be optimal to exclude some individuals from consuming this good.
} 
good facility of fixed size. ${ }^{9}$ Increasing the size of the facility shifts this curve outward. In this example, total consumption of the good increases as benefits accrue more evenly, so the good is not completely rivalous in consumption.

Efficiency in distribution may be incompatible with excluding any consumer from the benefits of a shared good. This possibility is illustrated in Figure 2. In this example, the curve shows the upper boundary of all distributions that are possible from a fixed-size facility, but only the distributions between $a$ and $b$ are efficient. The example of allocating a police force between two precincts discussed in the preceding section is an example of this kind of shared good. Even if the whole force is located in one precinct, the presence of police in a nearby area will deter some criminal activity in the other precinct.

With a pure public good, there is only one efficient distribution possible from a given sized facility, as illustrated in Figure 3. In the theory of pure public goods, the units in which public good production and consumption are measured are the same. Thus, if group $g$ supplies $y_{g m}$ units of the $m$ th public good, each consumer receives $y_{g m}$ units of consumption. This is indicated by the point $a$ in Figure 3. If exclusion is possible, as is assumed here, then any distribution is possible in which no consumer obtains more than $y_{g m}$ units of this good.

Private goods can also be modelled as a special kind of shared good. As with public goods, units of private good consumption are the same as units of production. Private goods exhibit complete rivalry in consumption. Thus, as illustrated in Figure 4, the boundary of the sharing technology has slope -1 for a fixed quantity of this good available for distribution. Most private goods are supplied by firms. In terms of the model presented here, such a firm has a dual role; it is both the producer of the good and the group that makes it available to consumers. Not all goods that exhibit complete rivalry in consumption are privately supplied. For example, a municipal parking lot operating at full capacity exhibits complete rivalry in its usage. ${ }^{10}$ It is natural to think of it as a shared good with private characteristics.

These examples demonstrate that by appropriate specification of the sharing technology, my model of shared goods is general enough to include pure public goods, private goods, and impure public goods that exhibit partial

\footnotetext{
${ }^{9}$ The efficient distributions for a given sized facility are those distributions that are not weakly vector dominated by any other feasible distribution.

${ }^{10}$ See Holtermann (1972) for a discussion of this kind of publicly-provided good.
} 
rivalry in consumption as special cases. Musgrave's (1969) model of impure public goods is also a special case. In Musgrave's model, individuals supply public goods. The person who supplies a unit of a public good receives a unit of consumption, but the other individuals only receive a fraction of a unit. Using the terminology introduced here, the groups providing shared goods facilities are individuals and the sharing technologies are linear. Many of the standard examples of consumption externalities have this structure. To cite but one example, the externality a gardener generates for his or her neighbour is due to the fact that the neighbour can see the garden, not because there is a concern for the gardener's well-being.

However, in spite of its flexibility, there are important classes of impure public goods that are not captured by my framework. For example, if private goods are needed to enjoy the benefits of a public facility, as in Sandmo (1973), the facility is not a shared good in the sense used here. By relaxing some of my restrictive assumptions, other kinds of impure public goods can be interpreted as being shared goods. The congestible public facilities considered by Inman (1971) and Hillman (1978) provide the same consumption to all users of the facility, but the amount of this consumption depends on the number of individuals using the facility, not just its size. This pattern of feasible consumptions can be modelled using a nonconvex sharing technology.

An individual may consume the same shared good from more than one source. For example, mosquito eradication programmes run by neighbouring jurisdictions benefit residents in both locations. For all $i \in \mathcal{I}$, the total consumption of the $m$ th shared good is

$$
s_{i m}=\sum_{g=1}^{G} s_{i g m} .
$$

Individuals consume both private goods and shared goods and only care about their own consumption of these goods. A consumption bundle for individual $i$ is a vector $\left(\mathbf{x}_{i}, \mathbf{s}_{i}\right)=\left(x_{i 1}, \ldots, x_{i K}, s_{i 1}, \ldots, s_{i M}\right)$. Individual $i$ 's preferences are represented by the utility function $U^{i}: \mathbb{R}_{+}^{K+M} \rightarrow \mathbb{R}$. $U^{i}$ is assumed to be continuously differentiable, strictly quasiconcave, and strictly increasing in each argument.

The aggregate endowment of the $k$ th private good is $\omega_{k}$. There are no endowments of shared goods.

The model of the economy is completed by specifying the materials bal- 
ance constraints for the private goods. For each $k \in \mathcal{K}$,

$$
\sum_{i=1}^{I} x_{i k}+\sum_{m=1}^{M} z_{m k} \leq w_{k}+\omega_{k} .
$$

In other words, for the $k$ th private good, the amount allocated to individuals for consumption plus the amount allocated to firms as inputs for the production of shared goods cannot exceed the sum of the quantity produced and the endowment of this good.

An allocation for this economy consists of a consumption bundle $\left(\mathbf{x}_{i}, \mathbf{s}_{i}\right)$ for all $i \in \mathcal{I}$, a shared good production vector $\left(\mathbf{z}_{m}, y_{m}\right)$ for all $m \in \mathcal{M}$, a vector $\left(\mathbf{s}_{g m}, y_{g m}\right)$ for all $g \in \mathcal{G}$ and all $m \in \mathcal{M}$ describing the quantity of the $m$ th shared good facility owned by the $g$ th group and how its benefits are shared by consumers, and a private goods production vector w. An allocation is feasible if the shared good production constraint (1) is satisfied for all $m \in \mathcal{M}$, the constraint on the production of private goods (2) is satisfied, the sharing technology constraint (4) is satisfied for all $g \in \mathcal{G}$ and all $m \in \mathcal{M}$, the materials balance constraint for a shared good facility (3) is satisfied for all $m \in \mathcal{M}$, the materials balance constraint (5) for shared goods consumption is satisfied for all $i \in \mathcal{I}$ and all $m \in \mathcal{M}$, and the materials balance constraint for private goods (6) is satisfied for all $k \in \mathcal{K}$. $^{11}$

In order to facilitate the comparison of the optimality conditions for shared goods with those for public and private goods, various simplifying assumptions have been made. A number of generalizations of the model are possible. A shared good facility could produce more than one kind of consumption benefit. For example, firemen are often trained to provide emergency assistance to individuals who have suffered a heart attack in addition to being trained to put out fires. In the model presented in this section, each shared good has been identified with a single shared good facility. However, many shared goods facilities are in fact multidimensional. A fire department consists of various kinds of personnel, fire halls, fire trucks, etc. I have dichotomized the production of shared goods facilities from the sharing of the corresponding consumption benefits. In a more general model, the individual consumptions of a shared good could be expressed as a direct function of the private good inputs, without explicitly having a shared good facility as an intermediate product. Shared goods could also be used as inputs in

\footnotetext{
${ }^{11}$ There are also nonnegativity constraints on the $\mathbf{x}_{i}, \mathbf{s}_{i}, \mathbf{z}_{m}, y_{m}, \mathbf{s}_{g m}$, and $y_{g m}$. These constraints are not explicitly considered in the subsequent discussion.
} 
the production of other goods or, as in Sandmo (1974), private goods could appear as additional inputs in the sharing technology.

\section{Pareto Optimality}

In this section, I present and interpret the necessary conditions for Pareto optimality for the economy described in the preceding section when complete variability in the consumption shares is possible. That is, for all $g \in \mathcal{G}$, all $m \in \mathcal{M}$, all positive $y_{g m}$, and all strictly positive vectors $\mathbf{s}_{g m}$, there is an efficient distribution $\overline{\mathbf{s}}_{g m}$ of this shared good that is proportional to $\mathbf{s}_{g m}$. It is further assumed that the sharing function $S^{g m}(\cdot)$ is continuously differentiable. These assumptions exclude public goods and shared goods with limited variability in consumption shares, such as the good illustrated in Figure 2, from consideration. Fixed consumption shares are considered in the next section. For brevity, I only consider the necessary conditions for an interior optimum as it is straightforward to modify the analysis to take account of corner solutions.

Pareto optimal allocations are found by specifying target levels for utility (compatible with feasibility) for all but consumer 1 and choosing a feasible allocation to maximize consumer 1's utility subject to these utility constraints. For all $i \neq 1$, let $\bar{U}^{i}$ denote the target utility for consumer $i$. The utility constraint for consumer $i$ is then

$$
U^{i}\left(\mathbf{x}_{i}, \mathbf{s}_{i}\right) \geq \bar{U}^{i}
$$

Because the utility functions are increasing in all of their arguments, these constraints and all of the inequality constraints that define a feasible allocation hold with equality at a Pareto optimal allocation.

The Lagrangian for this optimization problem is

$$
\begin{array}{r}
\mathcal{L}=U^{1}\left(\mathbf{x}_{1}, \sum_{g=1}^{G} s_{1 g 1}, \ldots, \sum_{g=1}^{G} s_{1 g M}\right)-\sum_{i=2}^{I} \mu_{i}\left[\bar{U}^{i}-U^{i}\left(\mathbf{x}_{i}, \sum_{g=1}^{G} s_{i g 1}, \ldots, \sum_{g=1}^{G} s_{i g M}\right)\right] \\
-\sum_{m=1}^{M} \beta_{m} Y^{m}\left(\mathbf{z}_{m}, \sum_{g=1}^{G} y_{g m}\right)-\sum_{g=1}^{G} \sum_{m=1}^{M} \xi_{g m} S^{g m}\left(\mathbf{s}_{g m}, y_{g m}\right)-\gamma F(\mathbf{w}) \\
-\sum_{k=1}^{K} \lambda_{k}\left[\sum_{i=1}^{I} x_{i k}+\sum_{m=1}^{M} z_{m k}-w_{k}-\omega_{k}\right],
\end{array}
$$


where (3) (with equality) and (5) have been substituted into the production functions for shared goods facilities and into the utility functions so as to eliminate the variables $s_{i m}$ and $y_{m}$.

In addition to the satisfaction of all of the constraints with equality, the necessary conditions for an interior Pareto optimal allocation are:

$$
\begin{gathered}
\frac{\partial U^{1}}{\partial x_{1 k}}-\lambda_{k}=0, \quad \forall k \in \mathcal{K}, \\
\mu_{i} \frac{\partial U^{i}}{\partial x_{i k}}-\lambda_{k}=0, \quad \forall i \in \mathcal{I} \backslash\{1\}, \quad \forall k \in \mathcal{K}, \\
\frac{\partial U^{1}}{\partial s_{1 m}}-\xi_{g m} \frac{\partial S^{g m}}{\partial s_{1 g m}}=0, \quad \forall g \in \mathcal{G}, \quad \forall m \in \mathcal{M}, \\
\mu_{i} \frac{\partial U^{i}}{\partial s_{i m}}-\xi_{g m} \frac{\partial S^{g m}}{\partial s_{i g m}}=0, \quad \forall i \in \mathcal{I} \backslash\{1\}, \forall g \in \mathcal{G}, \quad \forall m \in \mathcal{M}, \\
-\beta_{m} \frac{\partial Y^{m}}{\partial z_{m k}}-\lambda_{k}=0, \quad \forall k \in \mathcal{K}, \quad \forall m \in \mathcal{M}, \\
-\beta_{m} \frac{\partial Y^{m}}{\partial y_{m}}-\xi_{g m} \frac{\partial S^{g m}}{\partial y_{g m}}=0,
\end{gathered}
$$

where, for compactness, the arguments of the functions that appear in (9)(15) have been suppressed.

From (9), (10), and (15), it follows that

$$
-\frac{\partial U^{i} / \partial x_{i k^{\prime}}}{\partial U^{i} / \partial x_{i k}}=-\frac{\partial F / \partial w_{k^{\prime}}}{\partial F / \partial w_{k}}, \quad \forall i \in \mathcal{I}, \forall k, k^{\prime} \in \mathcal{K} .
$$

This equation is the standard optimality condition for private goods. It says that for all individuals and for any pair of private goods, the marginal rate 
of substitution should equal the marginal rate of transformation. Of course, if the aggregate production set for private goods were disaggregated into production sets for individual firms, optimality would also require setting each firm's marginal rate of transformation equal to the aggregate marginal rate of transformation.

Private goods are also used in the production of shared goods facilities. For each pair of private goods, (13) and (15) imply that the marginal rate of transformation in the production of any shared good facility should be equal to the marginal rate of transformation in (16). Formally,

$$
-\frac{\partial Y^{m} / \partial z_{m k^{\prime}}}{\partial Y^{m} / \partial z_{m k}}=-\frac{\partial F / \partial w_{k^{\prime}}}{\partial F / \partial w_{k}}, \quad \forall k, k^{\prime} \in \mathcal{K}, \forall m \in \mathcal{M}
$$

Equations (9), (10), (11), and (12) imply that

$$
\begin{aligned}
-\frac{\partial U^{i^{\prime}} / \partial s_{i^{\prime} m}}{\partial U^{i^{\prime}} / \partial x_{i^{\prime} k}}=-\frac{\partial U^{i} / \partial s_{i m}}{\partial U^{i} / \partial x_{i k}} & \frac{\partial S^{g m} / \partial s_{i^{\prime} g m}}{\partial S^{g m} / \partial s_{i g m}} \\
& \forall i, i^{\prime} \in \mathcal{I}, \forall g \in \mathcal{G}, \forall k \in \mathcal{K}, \forall m \in \mathcal{M} .
\end{aligned}
$$

Before interpreting (18), it is useful to note that it implies that

$$
-\frac{\partial S^{g^{\prime} m} / \partial s_{i^{\prime} g^{\prime} m}}{\partial S^{g^{\prime} m} / \partial s_{i g^{\prime} m}}=-\frac{\partial S^{g m} / \partial s_{i^{\prime} g m}}{\partial S^{g m} / \partial s_{i g m}}, \quad \forall i, i^{\prime} \in \mathcal{I}, \forall g, g^{\prime} \in \mathcal{G}, \forall m \in \mathcal{M}
$$

Equation (19) shows that the marginal rate at which one person's consumption of a shared good can be transformed into another person's consumption of the same good, holding the size of the shared good facility and the other individuals' consumptions fixed, must be the same for all providers of this good. These marginal rates of transformation for the sharing technologies are measures of the rivalness in consumption. For a private good, this condition is automatically satisfied because the sharing technology's marginal rate of transformation is identically equal to -1 for any pair of consumers.

Equation (18) describes how the marginal rates of substitution between a shared good and a private good are related for different individuals at a Pareto optimal allocation. Specifically, for any pair of individuals, the marginal rate of substitution for the first individual should be set equal to the marginal rate of substitution for the second individual multiplied by the absolute value of the marginal rate at which the consumption of this 
shared good can be traded off between these two individuals in any group's sharing technology. ${ }^{12}$ For example, if this marginal rate of transformation is -3 (i.e., at the margin, it is optimal to substitute three units of the second person's consumption of this shared good for one unit of the first person's consumption), then the first person's marginal rate of substitution should be three times that of the second's. If the shared good is in fact a private good, because the sharing technology's marginal rates of transformation are then equal to $-1,(18)$ simply says that the individuals' marginal rates of substitution should be set equal to one another, which is the standard private goods optimality condition for consumption in (16).

It follows from (9)-(14) that

$$
\begin{aligned}
-\frac{\partial U^{i} / \partial s_{i m}}{\partial U^{i} / \partial x_{i k}}=-\frac{\partial S^{g m} / \partial s_{i g m}}{\partial S^{g m} / \partial y_{g m}} \cdot & \frac{\partial Y^{m} / \partial y_{m}}{\partial Y^{m} / \partial z_{m k}} \\
& \forall i \in \mathcal{I}, \forall g \in \mathcal{G}, \forall k \in \mathcal{K}, \forall m \in \mathcal{M} .
\end{aligned}
$$

The reciprocal of the first term on the right-hand-side of (20) is the marginal rate at which individual $i$ 's consumption of the $m$ th shared good can be increased by increasing the size of the corresponding shared good facility by the $g$ th group, holding everybody's else's consumption of this good fixed. An implication of (20) is that these marginal products must be the same for all groups providing this shared good facility. That is,

$$
-\frac{\partial S^{g m} / \partial y_{g m}}{\partial S^{g m} / \partial s_{i g m}}=-\frac{\partial S^{g^{\prime} m} / \partial y_{g^{\prime} m}}{\partial S^{g^{\prime} m} / \partial s_{i g^{\prime} m}}, \quad \forall i \in \mathcal{I}, \forall g, g^{\prime} \in \mathcal{G}, \forall m \in \mathcal{M} .
$$

The right-hand-side of (20) can be thought of as the marginal rate of transformation between the $k$ th private good and $i$ 's consumption of the $m$ th shared good. In absolute value, this marginal rate of transformation is the reciprocal of the product of (a) the marginal rate at which $i$ 's consumption of the $m$ th shared good can be increased when the size of this shared good facility is increased and (b) the marginal product of the $k$ th private good in the production of the $m$ th shared good facility. For any individual $i,(20)$

\footnotetext{
${ }^{12}$ Buchanan (1966, p. 412) has an optimality condition for the special case in which there are two individuals, one shared good, and one private good that is essentially the same as (18). After stating this condition, Buchanan went on to say that extending the analysis to more individuals and goods is 'formidable', but his argument as to why he thinks this is so is not very clear. Nothing analogous to (18) appears in the discussion of impure public goods in Buchanan (1968).
} 
says that the marginal rate of substitution between a shared good and a private good should equal this marginal rate of transformation.

In the special case in which the shared good is a private good, no distinction is made between the size of the facility and the total consumption of this good. Increasing the quantity of the shared good available increases $i$ 's consumption by the same amount when everybody else's consumption is unchanged, so the first term on the right-hand-side of (20) is equal to -1 . Equation (20) then says that the marginal rate of substitution between this good and any other private good for any consumer should be equal to the marginal rate of transformation. ${ }^{13}$ Hence, the standard optimality condition for a pair of private goods given in (16) is implied by (18) and (20) when the shared good exhibits complete rivalry in consumption.

\section{Consumption Shares}

In the preceding section, by reducing the consumption of a shared good by one individual, it was possible to increase the consumption of this good for any other individual. This is not feasible with a pure public good. For this reason, the analysis in Section 3 is not able to shed light on how the optimality conditions for shared goods are related to Samuelson's (1954) optimality condition for public goods. In this section, I reformulate the model in terms of the fraction of the aggregate consumption of a shared good allocated to each individual and use this alternative description of the sharing technologies to derive necessary conditions for Pareto optimality for a shared goods economy. These conditions provide an alternative perspective on the properties of Pareto optimal allocations from that of the preceding section. In particular, the consumption shares version of the model naturally leads to a condition that characterizes the optimal scale of production of a shared good facility for fixed consumption shares, from which the optimality condition for public goods follows as a special case. I also show that the optimality conditions for private goods are a special case of the optimality conditions obtained using this version of the model. ${ }^{14}$ In order to focus on the new insights provided by modelling sharing technologies using consumption

\footnotetext{
${ }^{13}$ When comparing (16) and (20), it should be borne in mind that input quantities are negative in (16), but they are positive in (20).

${ }^{14}$ In Weymark (1979), I also modelled sharing technologies in terms of consumption shares, but only considered the polar cases of public and private goods.
} 
shares, I simplify the description of the economy by assuming that each shared good is only provided by one group. As in Section 3, I only consider the necessary conditions for an interior optimum.

The total consumption of the $m$ th shared good is

$$
s_{m}=\sum_{i=1}^{I} s_{i m} .
$$

Letting $a_{i m}$ denote person $i$ 's share of this total, $s_{i m}$ can be written as

$$
s_{i m}=a_{i m} s_{m}
$$

The vector of nonnegative consumption shares for the $m$ th shared good is $\mathbf{a}_{m}=\left(a_{1 m}, \ldots, a_{I m}\right)$, where

$$
\sum_{i=1}^{I} a_{i m}=1 .
$$

Using these shares, the sharing technology $\bar{S}^{m}$ for this good is the set of $\left(s_{m}, \mathbf{a}_{m}, y_{m}\right)$ for which

$$
\bar{S}^{m}\left(s_{m}, \mathbf{a}_{m}, y_{m}\right) \leq 0
$$

where $\bar{S}^{m}(\cdot)$ is the sharing function corresponding to $\bar{S}^{m}$. To be consistent with the original formulation of the model, we must have

$$
\bar{S}^{m}\left(s_{m}, \mathbf{a}_{m}, y_{m}\right)=S^{m}\left(a_{1 m} s_{m}, \ldots, a_{I m} s_{m}, y_{m}\right),
$$

where $S^{m}(\cdot)$ is the function that characterizes the sharing technology for the $m$ th shared good when the technology is expressed in terms of levels of consumption. In addition to the properties that $\bar{S}^{m}$ inherits from $S^{m}$ (i.e., the properties assumed for $S^{g m}$ in Section 2), $\bar{S}^{m}(\cdot)$ is assumed to be continuously differentiable. If the shares can't be varied, as would be the case with a pure public good, this differentiability assumption only applies to $s_{m}$ and $y_{m}$.

An allocation is defined as in Section 2 except that now there is only one vector $\left(s_{m}, \mathbf{a}_{m}, y_{m}\right)$ describing the consumption of the $m$ th shared good, rather than a separate sharing vector $\left(\mathbf{s}_{g m}, y_{g m}\right)$ for each group. To define the set of feasible allocations, (23)-(25) replace (4) and the materials balance 
constraints (3) and (5) are omitted because there is only one group for each shared good. ${ }^{15}$

The Lagrangian used to find necessary conditions for Pareto optimality for this version of the model is

$$
\begin{gathered}
\overline{\mathcal{L}}=U^{1}\left(\mathbf{x}_{1}, \mathbf{s}_{1}\right)-\sum_{i=2}^{I} \mu_{i}\left[\bar{U}^{i}-U^{i}\left(\mathbf{x}_{i}, \mathbf{s}_{i}\right)\right]-\sum_{m=1}^{M} \beta_{m} Y^{m}\left(\mathbf{z}_{m}, y_{m}\right) \\
-\sum_{m=1}^{M} \xi_{m} \bar{S}^{m}\left(s_{m}, \mathbf{a}_{m}, y_{m}\right)-\sum_{i=1}^{I} \sum_{m=1}^{M} \rho_{i m}\left[s_{i m}-a_{i m} s_{m}\right] \\
-\sum_{m=1}^{M} \phi_{m}\left[\sum_{i=1}^{I} a_{i m}-1\right]-\gamma F(\mathbf{w}) \\
-\sum_{k=1}^{K} \lambda_{k}\left[\sum_{i=1}^{I} x_{i k}+\sum_{m=1}^{M} z_{m k}-w_{k}-\omega_{k}\right] .
\end{gathered}
$$

Pareto optimality requires that all of the constraints described above hold with equality. In addition, when there are no constraints on the consumption shares other than the adding-up constraint (24), the necessary conditions for an interior Pareto optimum allocation are: (9), (10), (13), (15),

$$
\begin{gathered}
\frac{\partial U^{1}}{\partial s_{1 m}}-\rho_{1 m}=0, \quad \forall m \in \mathcal{M}, \\
\mu_{i} \frac{\partial U^{i}}{\partial s_{i m}}-\rho_{i m}=0, \quad \forall i \in \mathcal{I} \backslash\{1\}, \quad \forall m \in \mathcal{M}, \\
-\beta_{m} \frac{\partial Y^{m}}{\partial y_{m}}-\xi_{m} \frac{\partial \bar{S}^{m}}{\partial y_{m}}=0, \quad \forall m \in \mathcal{M}, \\
-\xi_{m} \frac{\partial \bar{S}^{m}}{\partial s_{m}}+\sum_{i=1}^{I} \rho_{i m} a_{i m}=0, \quad \forall m \in \mathcal{M},
\end{gathered}
$$

\footnotetext{
${ }^{15}$ Because the right-hand-side of (23) has a multiplicative form, the set of feasible allocations need not be convex. Hence, the necessary conditions for Pareto optimality obtained using this model need not be sufficient. A similar problem arises in stock market economies. See Drèze $(1972,1974)$.
} 


$$
-\xi_{m} \frac{\partial \bar{S}^{m}}{\partial a_{i m}}+\rho_{i m} s_{m}-\phi_{m}=0, \quad \forall i \in \mathcal{I}, \forall m \in \mathcal{M}
$$

The last equation does not apply to any shared good whose consumption shares cannot be varied. Goods with fixed consumption shares are called quasi-public goods in Mohring and Boyd (1971). A pure pure public good is a quasi-public good whose consumption shares are all equal to $1 / I$.

As in the preceding section, the standard optimality conditions for private goods, (16) and (17), follow from these first-order conditions. That is, for any pair of private goods, there must be equality between the marginal rates of substitution in consumption and the marginal rates of transformation in production (of private goods and shared goods facilities).

In the Appendix, it is shown that the conditions that characterize the optimal scale of production of the shared good facilities for given consumption shares are given by

$$
\sum_{i=1}^{I} a_{i m}\left[-\frac{\partial U^{i} / \partial s_{i m}}{\partial U^{i} / \partial x_{i k}}\right]=-\frac{\partial \bar{S}^{m} / \partial s_{m}}{\partial \bar{S}^{m} / \partial y_{m}} \cdot \frac{\partial Y^{m} / \partial y_{m}}{\partial Y^{m} / \partial z_{m k}}, \quad \forall k \in \mathcal{K}, \forall m \in \mathcal{M}
$$

The left-hand-side of (33) is the share-weighted sum of the marginal rates of substitution between the $m$ th shared good and the $k$ th private good using the vector $\mathbf{a}_{m}$ of consumption shares as weights. The reciprocal of the absolute value of the right-hand-side of (33) is

$$
\frac{\partial \bar{S}^{m} / \partial y_{m}}{\partial \bar{S}^{m} / \partial s_{m}} \cdot \frac{\partial Y^{m} / \partial z_{m k}}{\partial Y^{m} / \partial y_{m}}, \quad \forall k \in \mathcal{K}, \forall m \in \mathcal{M}
$$

The absolute value of the first term in this expression is the rate at which aggregate consumption of the $m$ th shared good increases with an increase in the size of the corresponding facility holding the consumption shares fixed at $\mathbf{a}_{m}$ and the absolute value of the second term is the marginal product of the $k$ th private good in the production of this facility. Hence, the absolute value of the right-hand-side of (33) is the reciprocal of the marginal rate at which the $k$ th private good can be transformed into aggregate consumption of the $m$ th shared good given the consumption shares $\mathbf{a}_{m}$ (i.e., the right-hand-side of (33) is the marginal rate of transformation between the $k$ th private good and the aggregate consumption of the $m$ th shared good). Note that while the marginal rates of substitution in (33) are expressed in terms of individual 
consumptions, the marginal rate of transformation is expressed in terms of aggregate consumption. ${ }^{16}$

With a pure public good, the consumption shares are all equal to $1 / I$. Further, the units in which production and consumption are measured are the same. Thus, increasing the size of a public good facility by one unit increases total consumption by $I$ units, which implies that the first term on the right-hand-side of (33) is equal to $1 / I$. Hence, if the $m$ th shared good is a pure public good, then

$$
\sum_{i=1}^{I}\left[-\frac{\partial U^{i} / \partial s_{i m}}{\partial U^{i} / \partial x_{i k}}\right]=\frac{\partial Y^{m} / \partial y_{m}}{\partial Y^{m} / \partial z_{m k}} .
$$

This equation is the optimality condition for public good provision due to Samuelson (1954). In words, the sum of the marginal rates of substitution between a public good and a private good should be set equal to their marginal rate of transformation in production.

When consumption shares are variable, the optimal consumptions of a shared good can be determined by simultaneously solving (i) the condition for the optimal facility size for fixed consumption shares and (ii) the conditions that characterize the optimal consumptions for fixed facility size. ${ }^{17}$ These conditions are given by (33) and (18), respectively.

Further insight into the properties of Pareto optimal allocations when shares are variable can be obtained by considering the implications of the first-order conditions in (32). In the Appendix, it is shown that if consumption shares are chosen optimally, then

$$
\begin{gathered}
{\left[\frac{\partial U^{i} / \partial s_{i m}}{\partial U^{i} / \partial x_{i k}}-\frac{\partial U^{i^{\prime}} / \partial s_{i^{\prime} m}}{\partial U^{i^{\prime}} / \partial x_{i^{\prime} k}}\right]=\left[\frac{\partial Y^{m} / \partial y_{m}}{\partial Y^{m} / \partial z_{m k}}\right]\left[\frac{\partial S^{m} / \partial s_{i m}}{\partial S^{m} / \partial y_{m}}-\frac{\partial S^{m} / \partial s_{i^{\prime} m}}{\partial S^{m} / \partial y_{m}}\right]} \\
\forall i, i^{\prime} \in \mathcal{I}, \forall k \in \mathcal{K}, \forall m \in \mathcal{M} .
\end{gathered}
$$

Note that the sharing technologies have been expressed in terms of individual levels of consumption in (36), as in Sections 2 and 3, rather than in terms of consumption shares.

\footnotetext{
${ }^{16}$ The optimality condition for quasi-public goods in Breton (1974) is a special case of (33).

${ }^{17}$ Buchanan $(1966,1968)$ has emphasized these two aspects of shared good provision. Gevers' (1974) optimality conditions for a stock market economy also include separate conditions for optimality in scale and in distribution.
} 
The expression

$$
\frac{\partial S^{m} / \partial y_{m}}{\partial S^{m} / \partial s_{i m}} \cdot \frac{\partial Y^{m} / \partial z_{m k}}{\partial Y^{m} / \partial y_{m}}
$$

is the marginal rate at which person $i$ 's consumption of the $m$ th shared good can be increased by increasing the amount of the $k$ th private good used in the production of the $m$ th shared good facility, holding everyone else's consumption of this good fixed. The reciprocal of (37) is thus the rate at which the input of the $k$ th private good must be increased in order to increase $i$ 's consumption of the $m$ th shared good, holding the quantities of the other inputs fixed (i.e., it is the absolute value of the marginal rate of transformation between the $k$ th private good and $i$ 's consumption of the $m$ th shared good). For any pair of individuals, (36) shows that the difference between their marginal rates of transformation must equal the difference between these individuals' marginal rates of substitution for these goods.

The standard optimality conditions for private goods follow from (33) and (37). If the $m$ th shared good is a private good, a one unit increase in the facility size is needed in order to increase any individual's consumption of this good by one unit. Hence, the right-hand-side of (36) is 0, which implies that the marginal rate of substitution between this good and any other private good must be the same for all individuals. This, in turn, implies that the left-hand-side of (33) is equal to this common marginal rate of substitution. Because an additional unit of a shared good increases total consumption by one unit when a shared good is a private good, the right-hand-side of (33) is the marginal rate of transformation between the two private goods.

\section{Concluding Remarks}

In this article, I have used sharing technologies to model the kinds of impure public goods considered by Buchanan $(1966,1967,1968)$. One of the virtues of the shared goods framework is that public and private goods are special cases obtained by appropriately specifying the sharing technology. However, there are important kinds of impure public goods that are not captured by my model. By relaxing some of my restrictive assumptions, sharing technologies can be used to describe other kinds of impure public goods. Some of the possibilities for generalizing the model have been described in Section 2 .

In deriving the optimality conditions, I have assumed that none of the inequality constraints bind. An implication of this assumption is that it 
is never optimal to exclude any individual from the consumption of any shared good. By taking into account the possibility that some nonnegativity constraints on consumption may bind, the optimality conditions also identify which individuals should consume each shared good.

Having identified necessary conditions for an allocation in a shared goods economy to be Pareto optimal, it is natural to investigate whether there are institutional arrangements that generate Pareto optimal outcomes. In Weymark (1977), I considered two alternative mechanisms for the production and distribution of shared goods whose equilibria are Pareto optimal. The first of these mechanisms is a generalization of the Lindahl mechanism for the allocation of public goods. In the second mechanism, the provision of shared goods is determined cooperatively, with private goods allocated using competitive markets. In this article, I have assumed that individuals can be costlessly excluded from the consumption of any shared good. Whether or not exclusion is possible has no bearing on the optimality conditions obtained here. However, the possibility of exclusion is an important factor in determining which institutional arrangements, if any, can support Pareto optimal outcomes.

\section{Appendix}

Let $\mu_{1}=1$. Multiplying (28) by $a_{1 m}$ and (29) by $a_{i m}$ and then summing the resulting equations over $i$ yields

$$
\sum_{i=1}^{I} a_{i m} \mu_{i} \frac{\partial U^{i}}{\partial s_{i m}}=\sum_{i=1}^{I} \rho_{i m} a_{i m}, \quad \forall m \in \mathcal{M} .
$$

Substituting (31) into (A.1), we obtain

$$
\sum_{i=1}^{I} a_{i m} \mu_{i} \frac{\partial U^{i}}{\partial s_{i m}}=\xi_{m} \frac{\partial \bar{S}^{m}}{\partial s_{m}}, \quad \forall m \in \mathcal{M}
$$

From (9), (10), and (13), it follows that

$$
\mu_{i} \frac{\partial U^{i}}{\partial x_{i k}}=-\beta_{m} \frac{\partial Y^{m}}{\partial z_{m k}}, \quad \forall i \in \mathcal{I}, \forall k \in \mathcal{K}, \forall m \in \mathcal{M} .
$$

Solving (30) for $-\beta_{m}$ and substituting in (A.3) yields

$$
\mu_{i} \frac{\partial U^{i}}{\partial x_{i k}}=\xi_{m} \frac{\partial \bar{S}^{m}}{\partial y_{m}}\left[\frac{\partial Y^{m} / \partial z_{m k}}{\partial Y^{m} / \partial y_{m}}\right], \quad \forall i \in \mathcal{I}, \forall k \in \mathcal{K}, \forall m \in \mathcal{M}
$$


From (A.2) and (A.4), it then follows that

$$
\begin{aligned}
& \sum_{i=1}^{I} a_{i m}\left[\frac{\mu_{i} \partial U^{i} / \partial s_{i m}}{\mu_{j} \partial U^{j} / \partial x_{j k}}\right]=\frac{\partial \bar{S}^{m} / \partial s_{m}}{\partial \bar{S}^{m} / \partial y_{m}} \cdot \frac{\partial Y^{m} / \partial y_{m}}{\partial Y^{m} / \partial z_{m k}}, \\
& \forall j \in \mathcal{I}, \forall k \in \mathcal{K}, \forall m \in \mathcal{M} .
\end{aligned}
$$

From (9) and (10), we also have that

$$
\mu_{i} \frac{\partial U^{i}}{\partial x_{i k}}=\mu_{j} \frac{\partial U^{j}}{\partial x_{j k}}, \quad \forall i, j \in \mathcal{I}, \forall k \in \mathcal{K} .
$$

Substituting (A.6) into (A.5) gives (33).

Equations (28), (29), and (32) imply that

$$
\begin{aligned}
&-\xi_{m} \frac{\partial \bar{S}^{m}}{\partial a_{i m}}+\mu_{i} s_{m} \frac{\partial U^{i}}{\partial s_{i m}}=-\xi_{m} \frac{\partial \bar{S}^{m}}{\partial a_{j m}}+\mu_{j} s_{m} \frac{\partial U^{j}}{\partial s_{j m}} \\
& \forall i, j \in \mathcal{I}, \quad \forall m \in \mathcal{M}
\end{aligned}
$$

or, equivalently,

$$
s_{m}\left[\mu_{i} \frac{\partial U^{i}}{\partial s_{i m}}-\mu_{j} \frac{\partial U^{j}}{\partial s_{j m}}\right]=\xi_{m}\left[\frac{\partial \bar{S}^{m}}{\partial a_{i m}}-\frac{\partial \bar{S}^{m}}{\partial a_{j m}}\right],
$$

$\forall i, j \in \mathcal{I}, \forall m \in \mathcal{M}$

From (9) and (10), it then follows that

$$
\begin{aligned}
\lambda_{k} s_{m}\left[\frac{\partial U^{i} / \partial s_{i m}}{\partial U^{i} / \partial x_{i k}}-\frac{\partial U^{j} / \partial s_{j m}}{\partial U^{j} / \partial x_{j k}}\right]=\xi_{m} & {\left[\frac{\partial \bar{S}^{m}}{\partial a_{i m}}-\frac{\partial \bar{S}^{m}}{\partial a_{j m}}\right], } \\
& \forall i, j \in \mathcal{I}, \forall k \in \mathcal{K}, \forall m \in \mathcal{M} .
\end{aligned}
$$

Using (13) and (30) to eliminate the multipliers from (A.9), we obtain

$$
\begin{array}{r}
s_{m}\left[\frac{\partial U^{i} / \partial s_{i m}}{\partial U^{i} / \partial x_{i k}}-\frac{\partial U^{j} / \partial s_{j m}}{\partial U^{j} / \partial x_{j k}}\right]=\left[\frac{1}{\partial \bar{S}^{m} / \partial y_{m}}\right]\left[\frac{\partial Y^{m} / \partial y_{m}}{\partial Y^{m} / \partial z_{m k}}\right]\left[\frac{\partial \bar{S}^{m}}{\partial a_{i m}}-\frac{\partial \bar{S}^{m}}{\partial a_{j m}}\right] \\
\forall i, j \in \mathcal{I}, \forall k \in \mathcal{K}, \forall m \in \mathcal{M} .
\end{array}
$$

From (26), it follows that

$$
\frac{\partial \bar{S}^{m}}{\partial a_{i m}}=s_{m} \frac{\partial S^{m}}{\partial s_{i m}}, \quad \forall i \in \mathcal{I}, \forall m \in \mathcal{M},
$$


and

$$
\frac{\partial \bar{S}^{m}}{\partial y_{m}}=\frac{\partial S^{m}}{\partial y_{m}}, \quad \forall m \in \mathcal{M} .
$$

Substituting (A.11) and (A.12) into (A.10), we obtain (36).

\section{References}

Breton, A. (1974), The Economic Theory of Representative Government, Chicago: Aldine.

Buchanan, J. M. (1965), An economic theory of clubs, Economica 32, 1-14.

Buchanan, J. M. (1966), Joint supply, externality and optimality, Economica 33, 404-415.

Buchanan, J. M. (1967), Breton and Weldon on public goods, Canadian Journal of Economics and Political Science 33, 111-115.

Buchanan, J. M. (1968), The Demand and Supply of Public Goods, Chicago: Rand McNally.

Cornes, R. and T. Sandler (1996), The Theory of Externalities, Public Goods, and Club Goods, Second Edition, Cambridge: Cambridge University Press.

Drèze, J. H. (1972), A tâtonnement process for investment under uncertainty, in G. P. Szegö and K. Shell, eds., Mathematical Methods in Investment and Finance, Amsterdam: North-Holland, 3-23.

Drèze, J. H. (1974), Investment under private ownership: Optimality, equilibrium and stability, in J. H. Drèze, ed., Allocation Under Uncertainty: Equilibrium and Optimality, London: Macmillan, 129-166.

Gevers, L. (1974), Competitive equilibrium of the stock exchange and Pareto efficiency, in J. H. Drèze, ed., Allocation Under Uncertainty: Equilibrium and Optimality, London: Macmillan, 167-191.

Hillman, A. L. (1978), The theory of clubs: A technological formulation, in A. Sandmo, ed., Essays in Public Economics, Lexington, Mass.: Lexington Books, 29-47.

Holtermann, S. E. (1972), Externalities and public goods, Economica 39, 78-87.

Inman, R. P. (1972), Towards an econometric model of local budgeting, Proceedings of the National Tax Association 64, 609-719.

Margolis, J. (1955), A comment on the pure theory of public expenditure, Review of Economics and Statistics 37, 347-349. 
Marshall, A. (1920), Principle of Economics, Eighth Edition, London: Macmillan.

Mohring, H. and J. H. Boyd (1971), Analyzing 'externalities': 'Direct interaction' vs. 'asset utilization' frameworks, Economica 38, 347-361.

Musgrave, R. A. (1969), Provision for social goods, in J. Margolis and H. Guitton, eds., Public Economics, London: Macmillan, 124-144.

Oakland, W. H. (1972), Congestion, public goods and welfare, Journal of Public Economics 1, 339-357.

Oakland, W. H. (1987), Theory of public goods, in A. J. Auerbach and M. Feldstein, eds., Handbook of Public Economics, Volume II, Amsterdam: North-Holland, 485-569.

Plott, C. R. and R. A. Meyer (1975), The technology of public goods, externalities, and the exclusion principle, in E. S. Mills, ed., Economic Analysis of Environmental Problems, New York: National Bureau of Economic Research/Columbia University Press, 65-90.

Samuelson, P. A. (1954), The pure theory of public expenditure, Review of Economics and Statistics 36, 387-389.

Sandmo, A. (1973), Public goods and the technology of consumption, Review of Economic Studies 40, 517-528.

Shoup, C. S. (1964), Standards for distributing a free governmental service: Crime protection, Public Finance/Finances Publiques 19, 383-392.

Tiebout, C. E. (1956), A pure theory of local public expenditures, Journal of Political Economy 64, 416-424.

Weymark, J. A. (1977), Essays in Public Economics, Ph.D. dissertation, Department of Economics, University of Pennsylvania.

Weymark, J. A. (1979), Optimality conditions for public and private goods, Public Finance Quarterly 7, 338-351. 


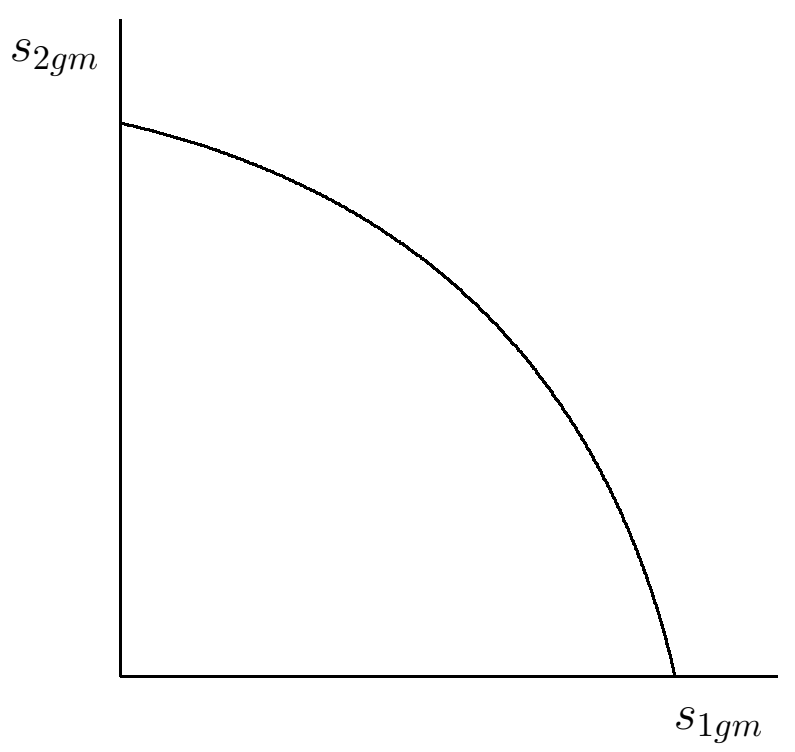

Figure 1

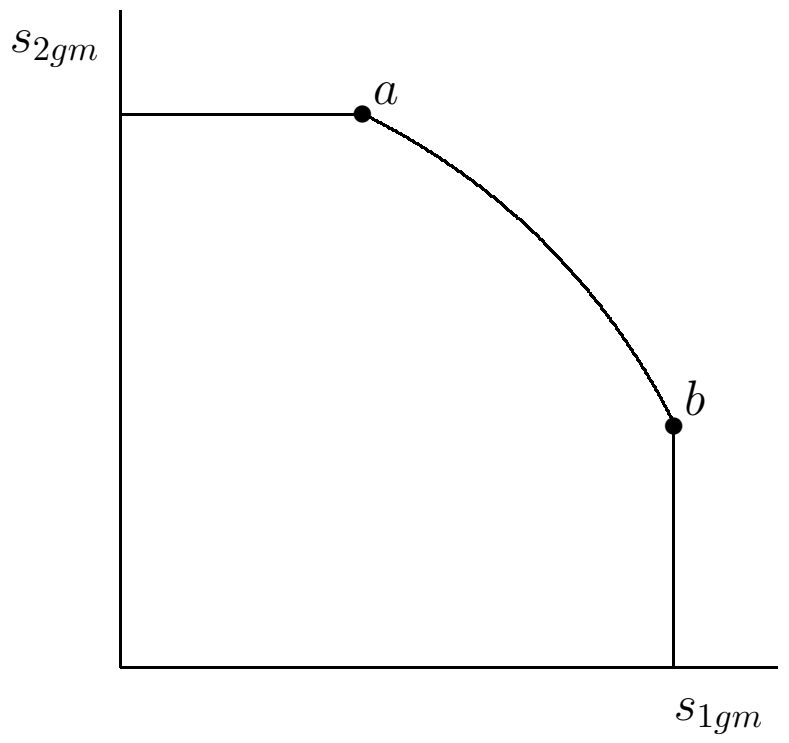

Figure 2 


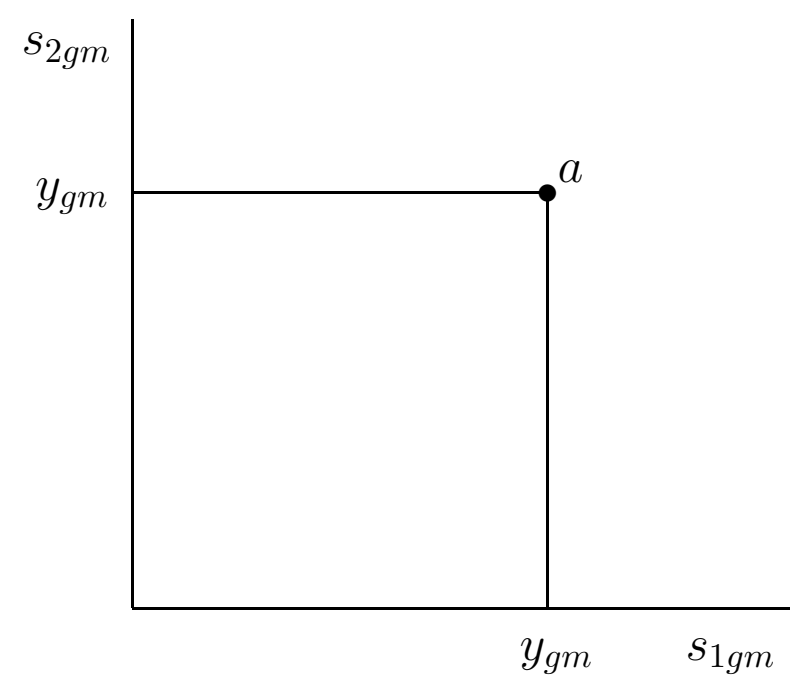

Figure 3

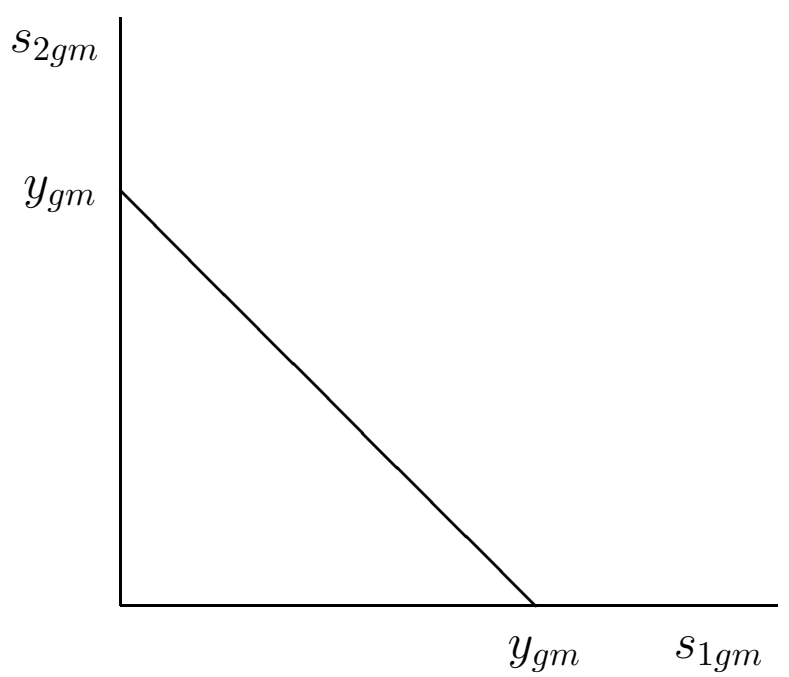

Figure 4 